EPJ Web of Conferences 64, 05009 (2014)

DOI: $10.1051 /$ epjconf/ 20146405009

(C) Owned by the authors, published by EDP Sciences, 2014

\title{
Oscillations of the Boundary Layer and High-frequency QPOs
}

\author{
A. A. Blinova ${ }^{1, a}$, M. Bachetti ${ }^{2}$, M. M. Romanova ${ }^{1}$ \\ ${ }^{1}$ Department of Astronomy, Cornell University, Ithaca, NY 14853-6801, USA \\ ${ }^{2}$ Institut de Recherche en Astrophysique et Planetologie, Toulouse, 31400, France
}

\begin{abstract}
We observed persistent high-frequency oscillations of the boundary layer near an accreting, weakly-magnetized star in global 3D MHD simulations. The tilted dipole magnetic field is not strong enough to open a gap between the star and the disk. Instead, it forms a highly-wrapped azimuthal field near the surface of the star which slows down rotation of the disk matter, while a small tilt of the field excites oscillations of the boundary layer with a frequency below the Keplerian frequency. This mechanism may be responsible for the high-frequency oscillations in accreting neutron stars, white dwarfs and classical T Tauri stars.
\end{abstract}

\section{Introduction}

Accretion through a boundary layer is expected in the cases where a star has a relatively weak magnetic field, so that the accretion disk reaches the surface of the star. In most investigations of the boundary layer the magnetic field has been neglected (e.g. [1-5]).

It is important to study the boundary layer in situations where a weak magnetic field is present. Paczyński suggested that the magnetic field may influence the dynamics of the boundary layer, forming a relatively thick torus [5]. He suggested that the high-frequency oscillations in the Dwarf Novaetype white dwarfs may be connected with oscillations of the weakly-magnetized, rotating torus [5]. The weak magnetic field may also influence boundary layer accretion in young stars, and determine the highest-frequency QPOs in accreting neutron stars in Low-mass X-ray Binaries (LMXBs) [6].

Global 3D MHD simulations of accretion to a star with a weak magnetic field have shown that even a small magnetosphere influences the dynamics of the inner disk [7]. Accretion in the boundary layer regime has also been studied in the cases where accretion in the disk is driven by the magnetorotational (MRI) instability [8,9]. Simulations have shown that a star's weak magnetic field is enhanced due to the rotation of matter in the inner disk, and this field influences boundary layer accretion.

The goal of our research was to perform long-lasting 3D MHD simulations of the boundary layer in weakly-magnetized stars, and to investigate possible high-frequency variabilities connected with this type of accretion. In our simulations we discovered high-frequency, quasi-periodic oscillations (QPOs) of the boundary layer. Such oscillations are expected in different types of stars. Here, we show an example of boundary layer oscillations in accreting neutron stars.

ae-mail: alisablinova@gmail.com

This is an Open Access article distributed under the terms of the Creative Commons Attribution License 2.0, which permits unrestricted use, distribution, and reproduction in any medium, provided the original work is properly cited. 


\section{Model}

To investigate the boundary layer, we performed multiple 3D MHD (magnetohydrodynamic) simulations using a Godunov-type (e.g. [10]) numerical code, written in a "cubed sphere" coordinate system rotating with the star [11]. We solved 8 partial differential equations for density, entropy, three components of momentum and three components of the magnetic field. Simulations were performed using a high grid resolution ( 244 grid points in the azimuthal direction). Initial and boundary conditions are described, e.g., in [8].

We took the mass of a simulated neutron star to be $M_{\star}=1.4 M_{\odot}$, dipole magnetic field $B=2 \times 10^{7} G$, and radius of the star $R_{\star}=10 \mathrm{~km}$. We considered accretion from an $\alpha$-disk [12] with $\alpha$-parameter $\alpha=0.04$. We used the Nowak-Wagoner potential [13] to approximate the general relativistic effects:

$$
\Phi_{\mathrm{NW}}=-\frac{G M_{\star}}{r}\left[1-3 \frac{G M_{\star}}{r c^{2}}+12\left(\frac{G M_{\star}}{r c^{2}}\right)^{2}\right]=-\frac{G M_{\star}}{r}\left[1-\frac{3 r_{g}}{2 r}+3\left(\frac{r_{g}}{r}\right)^{2}\right],
$$

where $r_{g}=2 G M_{\star} / c^{2}=4.15 \mathrm{~km}$ is the Schwarzschild radius. This potential gives a reasonable approximation of general relativistic effects near the star (better, e.g., than the Paczyński-Wiita potential [14]).

\section{Results}

From our simulations we observed a boundary layer which is relatively thick, $(0.05-0.1) R_{\star}$, due to the interaction of the inner disk matter with the weak magnetic field. Our simulations show persistent, high-frequency quasi-periodic oscillations (QPOs) of matter in the boundary layer. Fig. 1 (right four panels) shows an example of variation in the density distribution of the boundary layer over four consecutive moments in time. A density enhancement is formed and rotates with sub-Keplerian frequency. Highly-wrapped azimuthal field lines are formed in the inner disk, and are responsible for the relatively high thickness of the boundary layer (see Fig. 1, left two panels).

To find the frequency of the oscillations, we performed different analyses. Fig. 2 (left panel) shows a spot-omega diagram (see, e.g., [15]) of density distribution in the boundary layer, where the slope of the lines represents the frequency of the QPOs: $v \approx 1,250 \mathrm{~Hz}$. The Power Spectral Density analysis has shown the presence of a one-armed $(\mathrm{m}=1)$ density wave rotating at the same frequency (see Fig. 2, right panel). This frequency is lower than the Keplerian frequency at the surface of the star $\left(v_{\mathrm{Kep}} \approx 2,200 \mathrm{~Hz}\right)$, but is much higher than the frequency of the $\operatorname{star}\left(v_{\star}=186 \mathrm{~Hz}\right)$. This frequency is located below the maximum in the $\Omega_{\text {disk }}$ distribution, and therefore the observed wave may represent a radially-trapped density wave [16].

The frequency of $v \approx 1,250 \mathrm{~Hz}$ obtained in our simulations should be corrected, taking into account the fact that we used the Nowak-Wagoner potential instead of the exact GR approach. In the case of a Keplerian disk (no magnetic field), the frequency $v_{\mathrm{NW}}$ is different from the Keplerian frequency $\nu_{\mathrm{Kep}}=(1 / 2 \pi)\left(G M_{\star} / r^{3}\right)^{1 / 2}$, and is: $\nu_{\mathrm{NW}}=\nu_{\mathrm{Kep}}\left(1-3 \delta+9 \delta^{2}\right)^{1 / 2}$, where $\delta=r_{g} / r$. However, in Schwarzschild geometry, the frequency $v_{S}=v_{\text {Kep }}$ exactly. Taking into account the correction $v_{S}=v_{\mathrm{NW}}\left(1-3 \delta+9 \delta^{2}\right)^{-1 / 2}$ at a typical boundary layer radius of $r=1.1 R_{\star}$, we obtain $v_{S}=1,170 \mathrm{~Hz}$. On the other hand, a star has a spin $v_{\star}=186 \mathrm{~Hz}$, and a small correction for the Kerr geometry will slightly increase the frequency of boundary layer oscillations [17].

The boundary layer is adjacent to the star, however, the density wave in the boundary layer rotates much more rapidly than the star. The density distribution in the boundary layer right above the surface of the star reflects the propagation of this density wave. We observe one large spot which rotates with the frequency of the boundary layer (see Fig. 3, left two panels). Also, we often see several smaller 

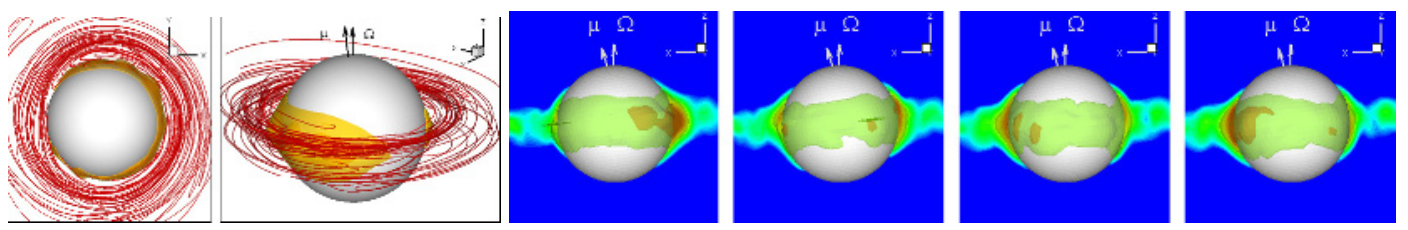

Figure 1. Left two panels: highly-wrapped azimuthal field lines in the inner disk. Right four panels: 3D side views of boundary layer oscillations, shown over four consecutive moments in time. Images are shown in a coordinate system rotating with the star.
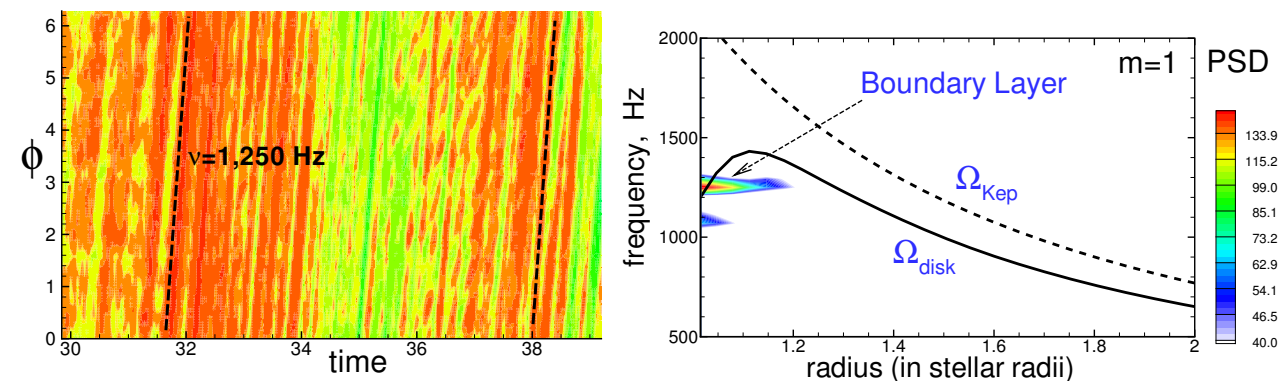

Figure 2. Left panel: a spot-omega diagram [15] of density distribution in the equatorial plane over $\phi$ (azimuthal direction) at the radius of $r=1.1 R_{\star}$ for different moments in time. Time is given in the units of one orbital period. The slope of the lines represents the frequency of boundary layer oscillations. Right panel: Power Spectral Density (PSD) is calculated for the surface density distribution near the star. The $\mathrm{m}=1$ (one-armed) density wave dominates and has a frequency of $v \approx 1,250 \mathrm{~Hz}$.

spots in the boundary layer (see Fig. 3, right two panels). These spots are probably connected with the Kelvin-Helmholtz instability, which could arise due to the velocity shear between the rapidly-rotating boundary layer and the slower-rotating layers above the surface of the star. We suggest that the observed boundary layer oscillations may be excited by the small tilt $\left(\Theta=5^{\circ}\right)$ of the magnetosphere. Such a tilt is expected in many realistic systems.

The highest frequencies of QPOs observed in LMXBs are $\sim 1,000-1,250 \mathrm{~Hz}$ [6]. In some models, it is suggested that these frequencies are associated with Keplerian frequency $v_{\mathrm{ISCO}}=$ $1,564\left(M_{\star} / 1.4 M_{\odot}\right)^{1 / 2}\left(R_{\star} / 10 \mathrm{~km}\right)^{-3 / 2} \mathrm{~Hz}$ at the last stable orbit $r_{\mathrm{ISCO}}=3 r_{g}=12.45\left(M_{\star} / 1.4 M_{\odot}\right) \mathrm{km}$, which gives a possible way of deriving the mass to radius ratio for neutron stars $[18,19]$. In our research, we show that in the presence of a weak magnetic field the rotation near a star is sub-Keplerian, and that the highest-frequency QPOs may instead be connected with the oscillations in the boundary layer.

\section{Summary}

We ran 3D MHD simulations of accretion to a weakly-magnetized neutron star of mass $M_{\star}=1.4 M_{\odot}$ and radius $r=10 \mathrm{~km}$, accreting in the boundary layer regime. We found persistent high-frequency quasi-periodic oscillations of the boundary layer which are associated with the propagation of a onearmed density wave. The frequency of these oscillations, $v \approx 1,170 \mathrm{~Hz}$, is lower than the Keplerian frequency at $R_{\star}$ and at $r_{\text {ISCO }}$. These oscillations should be systematically studied for different parameters of stars and their magnetic fields. 

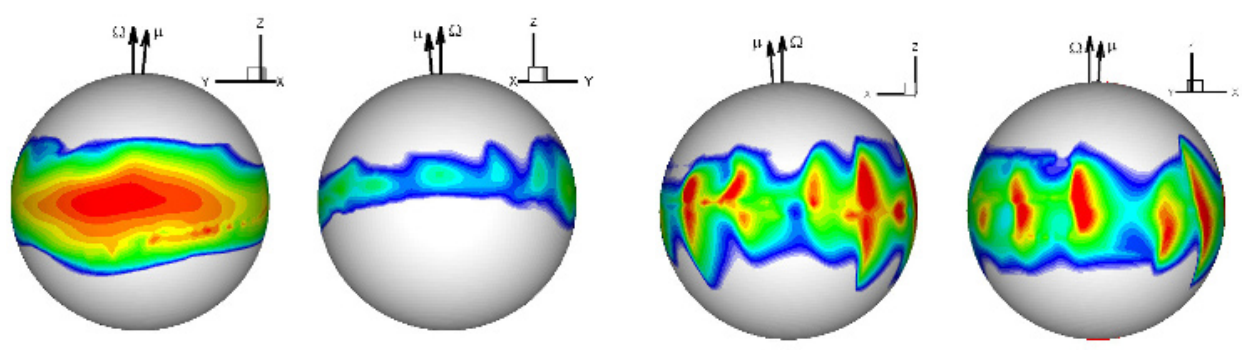

Figure 3. Left two panels: the dominant spot of the boundary layer at time $\mathrm{t}=40$, where time is measured in periods of Keplerian rotation at $r=1 \approx 3 R_{\star}$. The star is shown at two different angles. Right two panels: Two examples (at two different moments in time) of smaller spots in the boundary layer, which may be connected with the Kelvin-Helmholtz instability.

\section{Acknowledgment}

We thank the conference organizers, especially the chair, Dr. E. Bozzo, for the excellent meeting. Resources supporting this work were provided by the NASA High-End Computing (HEC) Program through the NASA Advanced Supercomputing (NAS) Division at Ames Research Center and the NASA Center for Computational Sciences (NCCS) at Goddard Space Flight Center. The research was supported by NASA grant NNX11AF33G and NSF grant AST-1008636.

\section{References}

[1] Popham R., Narayan R., Kenyon S. J., Hartmann L., Revista Mexicana de Astronomia y Astrofisica Conference Series 1, 229 (1995)

[2] Fisker J. L. and Balsara D. S., ApJ 635, L69 (2005)

[3] Inogamov N. A. and Sunyaev R. A., Astronomy Letters 25, 269 (1999)

[4] Piro A. L. and Bildsten L., Proceedings of ASP Conference 330, 197 (2005)

[5] Paczyński B. and Zytkow A., Polish Scientific Publ., p. 89 (1978)

[6] van der Klis M., Compact Stellar X-Ray Sources, Eds. Lewin W.H.G. and van der Klis M. (Cambridge Univ. Press, Cambridge, 2006) 39

[7] Romanova M. M. and Kulkarni A. K., MNRAS 398, 1105 (2009)

[8] Romanova M. M., Ustyugova G. V., Koldoba A. V., Lovelace R. V. E., MNRAS 421, 63 (2012)

[9] Balbus S. A. and Hawley J. F., ApJ 376, 214 (1991)

[10] Powell et al, J. Comp. Phys. 154, 284 (1999)

[11] Koldoba A. V., Romanova M. M., Ustyugova G. V., Lovelace R. V. E., ApJ 576, L53 (2002)

[12] Shakura N. I. and Sunyaev R. A., A\&A 24, 337 (1973)

[13] Nowak M. A. and Wagoner R. V., ApJ 378, 656 (1991)

[14] Abramowicz M. A., A\&A 500, 213 (2009)

[15] Kulkarni A. K. and Romanova M. M., MNRAS 386, 673 (2008)

[16] Lovelace R. V. E., Turner L., Romanova M. M., ApJ 701, 225 (2009)

[17] Miller M. C., Lamb F. K., Psaltis D., ApJ 508, 791 (1998)

[18] Boutelier M., Barret D., Miller M. C., MNRAS 399, 1901 (2009)

[19] Barret D., Bachetti M., Miller M. C., ApJ 728, 9 (2011) 